\title{
Did VV 29 collide with a dark Dark-Matter halo?
}

\author{
F. H. Briggs ${ }^{1}$, O. Möller ${ }^{1}$, J. L. Higdon ${ }^{1,2}$, N. Trentham ${ }^{3}$, and E. Ramirez-Ruiz ${ }^{3}$ \\ 1 Kapteyn Astronomical Institute, PO Box 800, 9700 AV Groningen, The Netherlands \\ 2 Department of Astronomy, Cornell University, Ithaca, NY 14853, USA \\ 3 Institute of Astronomy, Madingley Road, Cambridge CB3 0HA, UK
}

Received 27 August 2001 / Accepted 23 September 2001

\begin{abstract}
Westerbork Radio Synthesis Telescope observation of the galaxy VV $29=$ Arp $188=$ UGC 10214 shows that there are at least three distinct dynamical components whose kinematics can be traced in $21 \mathrm{~cm}$ line emission. The system appears to be the result of a galaxy-galaxy interaction. We identify a sufficient number of dynamical elements containing baryons (stars and neutral gas) that there is no compelling reason to postulate the presence of an additional dark matter halo that is devoid of detectable baryons. The central galaxy VV 29a is massive $\left(V_{\text {rot }} \sim 330 \mathrm{~km} \mathrm{~s}^{-1}\right)$ and gas rich $\left(M_{\mathrm{HI}} \sim 6 \times 10^{9} h^{-2} M_{\odot}\right)$. The distinctive optical plume (VV 29b), which extends eastward from the main galaxy, is also gas rich $\left(M_{\mathrm{HI}} \sim 3 \times 10^{9} h^{-2} M_{\odot}\right)$ and has a very low gradient in line of sight velocity $\left(<30 \mathrm{~km} \mathrm{~s}^{-1}\right)$ over $\sim 70 h^{-1} \mathrm{kpc}$. On the western side, there is an HI feature of $M_{\mathrm{HI}} \sim 4 \times 10^{8} h^{-2} M_{\odot}$ that participates strongly in orbital motion about the host in the same sense of rotation as the VV 29a itself. A blue, less massive, gas-rich galaxy "VV 29c" $\left(M_{\mathrm{HI}} \sim 9 \times 10^{8} h^{-2} M_{\odot}\right)$ appears clearly in the HI maps as an $\sim 170 \mathrm{~km} \mathrm{~s}^{-1}$ wide spectral feature, seen in projection against or, more likely, behind the west side of the host disk. Its high recessional velocity is counter to the host rotation direction. The optical images of Trentham et al. (2001) show signs of this blue dwarf against the redder VV 29a disk. The companion galaxy CGCG 27-021 = MGC 09-26-54 (at projected distance $\sim 115 h^{-1} \mathrm{kpc}$ ) is not detected in $21 \mathrm{~cm}$ line emission $\left(M_{\mathrm{HI}}<10^{9} h^{-2} M_{\odot}\right)$.
\end{abstract}

Key words. galaxies: dynamics - galaxies: evolution - galaxies: interaction - radio lines: galaxies

\section{Introduction}

The interacting system VV 29 (CGCG 275-023 = Arp $188=$ UGC 10214) has attracted recent attention for possibly being the victim of a collision with a pure dark matter halo (Trentham et al. 2001). This paper describes Westerbork Synthesis Radio Telescope observations that measure the neutral gas kinematics and show that there are numerous dynamical components in this interacting group that can be detected through the electromagnetic emission from their baryons.

The system is a member of several historical catalogs. The Atlas and Catalog of Interacting Galaxies (VorontsovVelyaminov 1959) listed the system as VV 29 and additionally assigned the label VV 29a to the bright spiral galaxy at the center of the system, as well as calling the faint optical feature to the east "VV 29b", as though it were a galaxy in its own right. Arp included the system in his Catalog of Peculiar Galaxies $(1962,1966)$, where the morphology of the thin optical plume VV 29b motivated its placement in the category of "Narrow Filaments". Arp added a comment that there is a disturbance inside the

Send offprint requests to: F. H. Briggs,

e-mail: fbriggs@astro.rug.nl western spiral arm of the host (VV 29a) and that "the filament may originate there". The new WSRT observation identifies this disturbance with a separate galaxy whose velocity is distinct from the host VV 29a and plume VV 29b components. We name the newly identified galaxy VV 29c. These three components are labeled on the images in Figs. 1 and 2.

A Nançay Radio Telescope detection of VV 29 provided a redshift of $9401 \pm 15 \mathrm{~km} \mathrm{~s}^{-1}$ (Bottinelli et al. 1993), but the WSRT observations show that the narrow emission feature in the Nançay spectrum emanates from the tidal plume component VV 29b. The new observation shows that the host galaxy VV 29a has a redshift $\sim 100 \mathrm{~km} \mathrm{~s}^{-1}$ less.

VV 29 finds itself a member of the poor cluster WBL 608 (White et al. 1999). Joseph et al. (1984) paired VV 29 with CGCG 27-021=MGC 09-26-54, an early type system with heliocentric redshift of $9439 \pm$ $27 \mathrm{~km} \mathrm{~s}^{-1}$ located at a projected distance of $115 h^{-1} \mathrm{kpc}$ (where is $h$ is the normalized Hubble parameter $h=$ $\left.H_{0} / 100 \mathrm{~km} \mathrm{~s}^{-1} \mathrm{Mpc}^{-1}\right)$.

The generation of long filamentary tails, counter-tails, and bridges in galaxies through tidal forces is well understood, thanks to concerted observational and theoretical 
efforts over the past three decades (for example, Toomre \& Toomre 1972; Schombert et al. 1990). In this view, VV 29 represents an ongoing merger between a massive spiral and one or more smaller companions, including one that may be partially obscured or disrupted as a result of the interaction. Similar interacting systems are common in the Arp Catalog. Blue tidal features such as the VV 29b plume are generally HI rich (Higdon \& Wallin 2001), making aperture synthesis observations in the $21 \mathrm{~cm}$ line extremely valuable in unraveling the interaction history of peculiar systems, and in revealing the presence of obscured or disrupted companion galaxies through their distinct kinematic components. For example, Hibbard \& van Gorkom (1996) presented extensive optical and HI observations of a merging sequence of galaxies (Toomre 1977) whose properties resemble the VV 29 system that we study here.

\section{The WSRT observations}

The Westerbork Synthesis Radio Telescope observed VV $29=$ UGC 10214 at celestial coordinates $16^{\mathrm{h}} 06^{\mathrm{m}} 03.9$, $55^{\circ} 25^{\prime} 32^{\prime \prime}$ (J2000) for 10 hours on 15-16 April 2001. Calibration scans on 3C 286 for 64 min preceding VV 29 and $3 \mathrm{C} 48$ for 23 min following VV 29 provided complex gain and passband calibrations. An adopted flux density of $14.94 \mathrm{Jy}$ for $3 \mathrm{C} 286$ sets the flux scale.

The spectrometer recorded 128 frequency channels in two linear polarizations to cover a $5 \mathrm{MHz}$ band centered on $z=0.031355$ in the Heliocentric reference frame. This provides a channel spacing of $8.5 \mathrm{~km} \mathrm{~s}^{-1}$. The first five channels (lowest redshift) and last eight channels showed deteriorating response, since they are close to the band edge; deleting them leaves a useful band covering a velocity range of $\sim 980 \mathrm{~km} \mathrm{~s}^{-1}$.

Application of standard calibration, mapping, and deconvolution steps in the Classic AIPS aperture synthesis imaging system led to a data cube of channel maps with synthesized beam resolution of $36^{\prime \prime} \times 26^{\prime \prime}$ (major axis at position angle $8^{\circ}$ ) with a ROBUST parameter of 3 in order to achieve an optimal sensitivity to weak emission. An unresolved continuum source (300 mJy) at $16^{\mathrm{h}} 06^{\mathrm{m}} 07^{\mathrm{s}} .6,55^{\circ} 21^{\prime} 35^{\prime \prime}(\mathrm{J} 2000)$ to the south of the target coordinates provides a secondary flux density calibration check as well as permitting refinement of the complex gain calibration through the course of the observation using self-calibration procedures. Model visibilities for the bright continuum source were subtracted from the UV dataset prior to construction of the cube of channel maps. Further continuum subtraction using linear interpolation between the extreme channels that were free of line emission was performed once the cube of channel maps had been computed. The surprisingly wide velocity spread of the HI associated with this system meant that there were few emission-free channels, and this adds some additional, systematic noise to the channel maps. The rms noise fluctuation in emission-free regions of the final channel maps was typically $\sim 0.33$ mJy per beam.

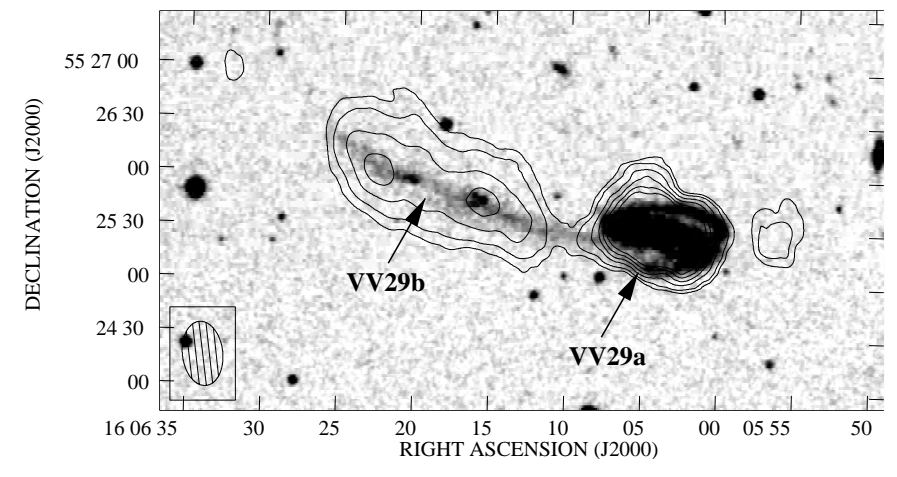

Fig. 1. VV 29a and VV 29b: integral HI column density contours for the velocity range -400 to $+240 \mathrm{~km} \mathrm{~s}^{-1}$ relative to $z=0.031355$ are superimposed on a DSS image at high contrast. This range includes the full velocity spread of the host galaxy VV 29a and the two plume components, while excluding the signal from the blue dwarf. The synthesized beam shape is shown at the lower left. Contour levels are 0.6, 1.2, 2.4, 3.6, $4.8,5.9 \times 10^{20} \mathrm{H}$ atoms $\mathrm{cm}^{-2}$.

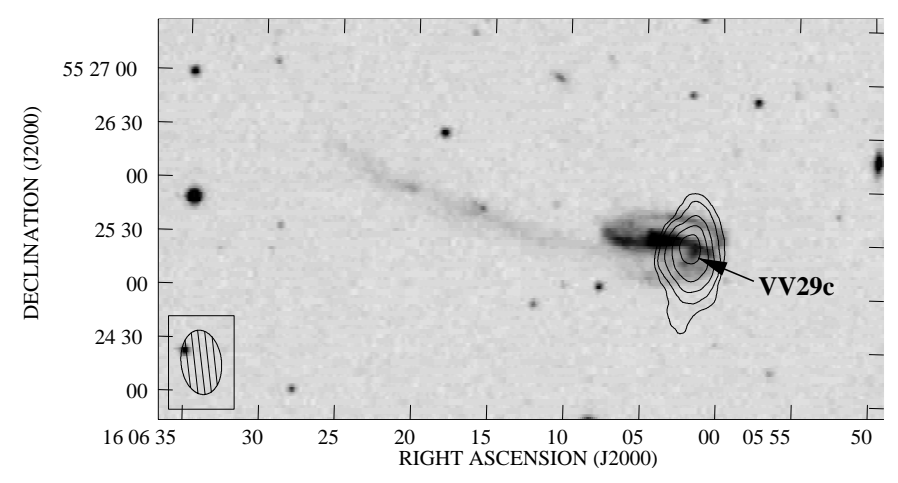

Fig. 2. VV 29c: integral HI column density contours for the velocity range +240 to $+470 \mathrm{~km} \mathrm{~s}^{-1}$ to accept the full range of the HI signal of the blue dwarf VV 29c are superimposed on the DSS image at low contrast. Contour levels are 0.6, 1.2, $2.4,3.6,4.8 \times 10^{20} \mathrm{H}$ atoms $\mathrm{cm}^{-2}$.

\section{Analysis}

Close inspection of the individual channel maps and cuts in distance-velocity planes revealed the nature of the principal HI features: 1. the host galaxy VV 29a itself, 2. a second galaxy of lower HI mass that is viewed along the same line of sight as the western side of the host, and 3. the eastern plume (detected in HI coincident with the distinctive optical plume). A possible counter-plume can be identified through the presence of faint HI emission on western side of the host. This section describes the observational evidence.

Figures 1 and 2 show contours of the integral HI emission overlaid on DSS images in order to show the correspondence between the extended HI emission and the optical plume and the location of the second gas-rich galaxy, VV 29c. Figures 3 and 4 show velocity contours corresponding to the Figs. 1 and 2, respectively. The velocity fields were obtained from the first moments of the HI profiles, after subjecting the images to automated thresholding computed from a spatially and spectrally smoothed 


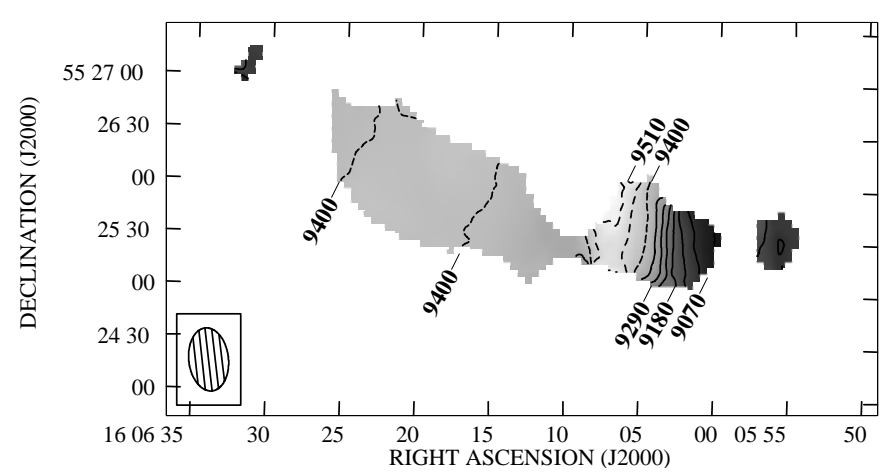

Fig. 3. VV 29a and VV 29b: velocity field computed from the first moment of the emission profiles for the gas in the velocity range -400 to $+240 \mathrm{~km} \mathrm{~s}^{-1}$ relative to $z=0.031355$. Contour levels are spaced at $55 \mathrm{~km} \mathrm{~s}^{-1}$ intervals.

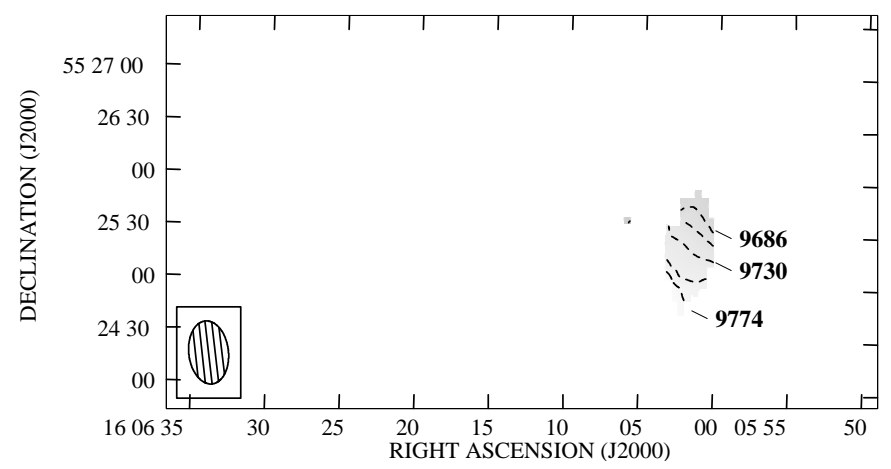

Fig. 4. VV 29c: velocity field computed from the first moment of the emission profiles for the gas in the velocity range +240 to $+470 \mathrm{~km} \mathrm{~s}^{-1}$ relative to $z=0.031355$. Lighter shading represents higher redshifted velocity. Contour levels are spaced at $22 \mathrm{~km} \mathrm{~s}^{-1}$ intervals.

version of the datacube. Figure 5 shows the integral spectral profiles for the components that can be seen in the maps.

Figures 1 to 5 make it clear that the galaxy itself is a large rotating disk system with a velocity spread of $\sim 700 \mathrm{~km} \mathrm{~s}^{-1}$; the western side of the galaxy has a projected velocity of approach along the line of sight, while the eastern side recedes. The systemic velocity falls at $9300 \mathrm{~km} \mathrm{~s}^{-1}$ (Heliocentric), which is $\sim 100 \mathrm{~km} \mathrm{~s}^{-1}$ less than the nominal redshift. There is a significant distortion of the $N_{\mathrm{HI}}$ contours extending northward at the eastern side of the galaxy. This distortion is coincident with very faint optical light in the deep images of Trentham et al. (2001) and is another symptom of severe gravitational interaction. A more detailed discussion of the features in the optical light follows in Sect. 4. The newly identified galaxy VV 29c has a systemic velocity of $9730 \mathrm{~km} \mathrm{~s}^{-1}$, and thus its motion would appear to carry it counter to the rotation direction of the host galaxy with a projected relative velocity of $\sim 430 \mathrm{~km} \mathrm{~s}^{-1}$. The rotational axis of VV $29 \mathrm{c}$ is oriented roughly perpendicular to the rotational axis of VV 29a.

Re-examination of the optical imaging from Trentham et al. (2001) gives an estimate of the ratio of optical $u$-band

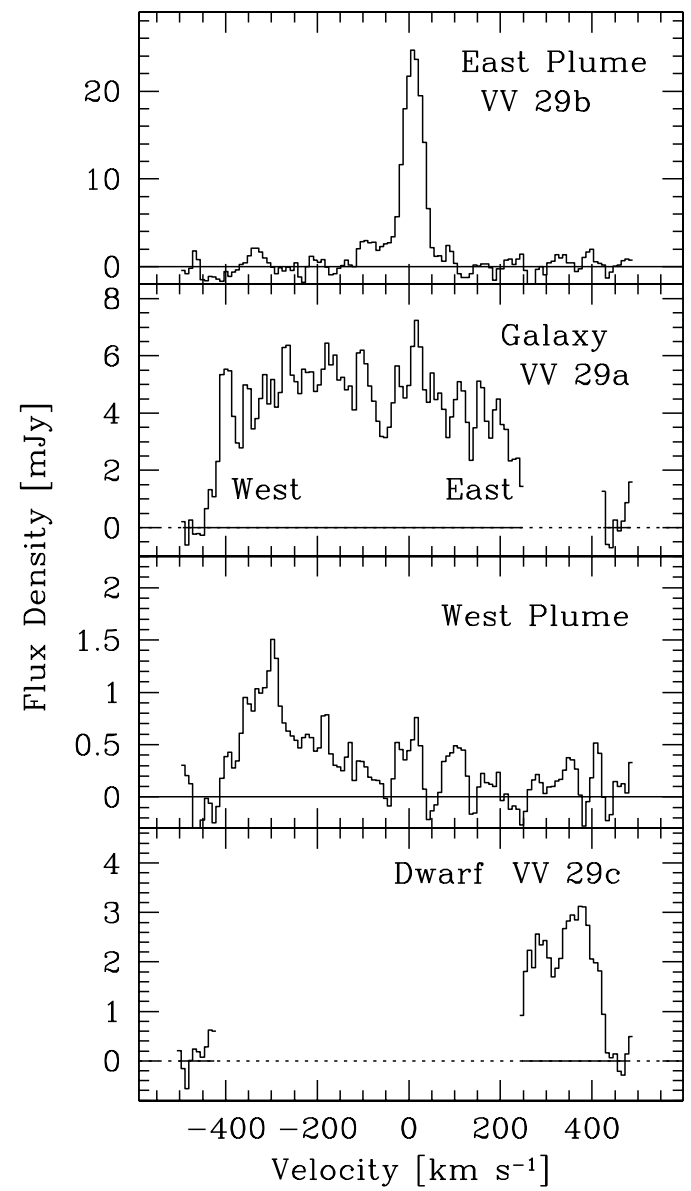

Fig. 5. Integral spectra for the four principal kinematic components obtained by summing pixels in the channel maps in the four regions. The zero velocity corresponds to $z=0.031355$ (Heliocentric).

light for VV 29c relative to VV 29a of 1:6, which is comparable to the ratio of approximately 1:7 in neutral hydrogen mass.

The plume to the east has very little velocity gradient along its length, falling at $\sim 100 \mathrm{~km} \mathrm{~s}^{-1}$ higher redshift than the systemic velocity of the host, and only near the base does the plume's velocity deviate toward the systemic velocity of the host. A clearer picture of the velocity structure comes from Fig. 6, the distance-velocity plane contour map oriented along the main body of the eastern plume. In this plot, the horizontal feature to the left is the constant velocity plume. The steeply sloped linear feature centered at position $\sim 0^{\prime \prime},-100 \mathrm{~km} \mathrm{~s}^{-1}$ is the host VV 29a, and the isolated feature to the upper right is a cut through VV 29c. The diffuse emission between the inner end of the plume and VV 29a represents a dispersed extension of the plume, which passes through zero relative velocity a little to the east of the center of VV 29a, and then picks up velocity in the sense of rotation of the host as it is traced further west. This could signify that the plume connects to the disk, possibly to one of the well defined spiral arms in the optical images. Figure 7 presents a distance-velocity cut that is aligned in an east-west direction along the major 


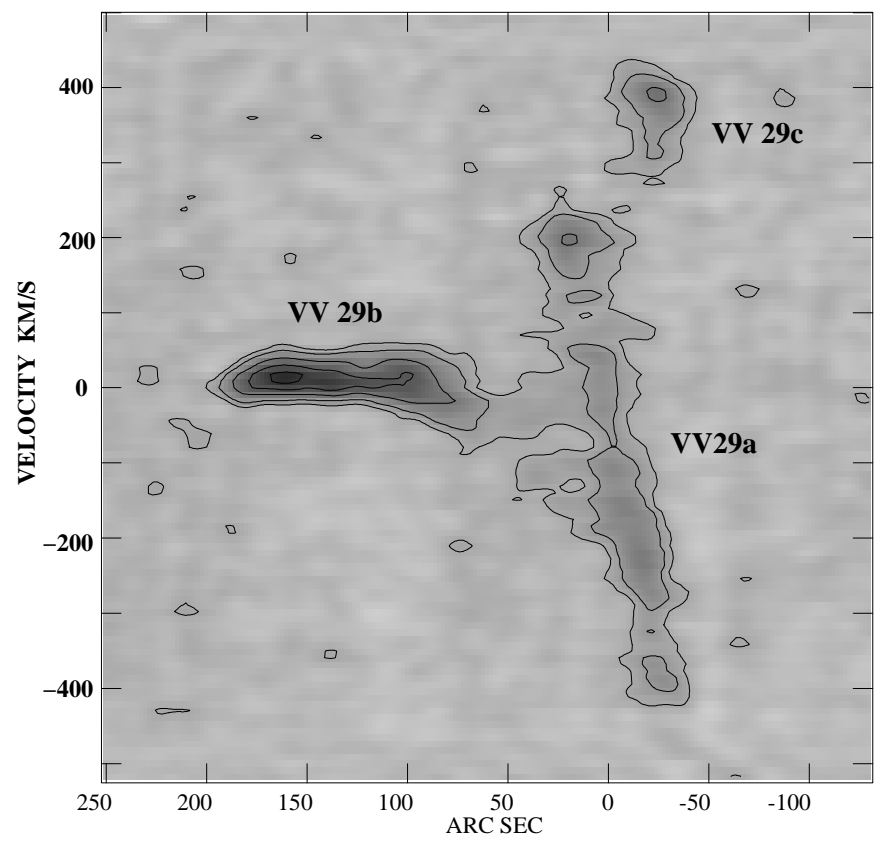

Fig. 6. Contours of HI brightness temperature in the distancevelocity plane along a line aligned with the eastern plume (at an angle of $18.5^{\circ}$ relative to a line of constant declination, corresponding to position angle $71.5^{\circ}$ ). The zero of velocity is $z=0.031355$, and the origin of distance falls at $16^{\mathrm{h}} 06^{\mathrm{m}} 04^{\mathrm{s}} .8$, $55^{\circ} 25^{\prime} 10^{\prime \prime}(\mathrm{J} 2000)$. Contour levels are 1, 2, 4, 6, $8 \mathrm{~K}$.

axis of the host, so that it cuts through the inner end of the long eastern plume and also the blob of emission seen on the western side of VV 29a in Fig. 1. One can smoothly extrapolate the rising speed of the inner edge of the plume to the western blob, which in turn has a projected velocity component along the line of sight in the sense of rotation of the host; however, we discuss later (in Sect. 4) the morphology of the faint optical emission from the western side of VV 29a, which leads us to associate the western blob with a counter-tail that is distinct from the VV 29b plume.

Figures 8 and 9 explore the degree of velocity collimation along the main body of the plume. Figure 8 shows spectra at three locations in the bright regions of the plume. Figure 9 shows the peak intensity, velocity centroid and velocity width $(F W H M)$ of Gaussian fits to the spectra as a function of distance along the plume. The velocity appears constant within $10 \mathrm{~km} \mathrm{~s}^{-1}$ over a distance of $\sim 40 h^{-1} \mathrm{kpc}$ along the plume. The velocity width $\sim 40 \mathrm{~km} \mathrm{~s}^{-1}\left(\sigma \approx 17 \mathrm{~km} \mathrm{~s}^{-1}\right)$ of the profiles is relatively wide in comparison to the velocity dispersions $\sim 6-10 \mathrm{~km} \mathrm{~s}^{-1}$ that characterize the HI in disk galaxies (van der Kruit \& Shostak 1982, 1984; Dickey et al. 1990; van Zee \& Bryant 1999).

A final distance-velocity plane cut in Fig. 10 along the major axis of the dwarf galaxy VV 29c (perpendicular to the major axis of the host VV 29a) shows the distinct velocity separation between the corotating gas in the disk on the west side of the host VV 29a (blue shifted) and the

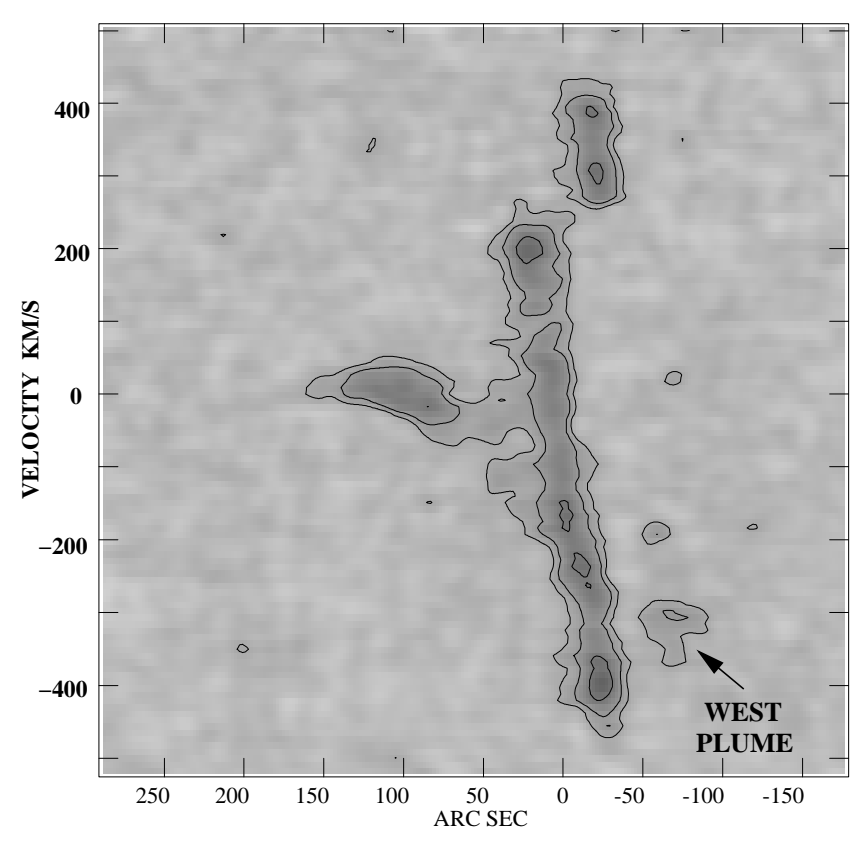

Fig. 7. Contours of HI brightness temperature in the distancevelocity plane along a line aligned with the major axis of the host galaxy VV 29a at position angle $90^{\circ}$. The zero of velocity is $z=0.031355$, and the origin of distance falls at galaxy center at $16^{\mathrm{h}} 06^{\mathrm{m}} 03^{\mathrm{s}} .9,55^{\circ} 25^{\prime} 32^{\prime \prime}(\mathrm{J} 2000)$.

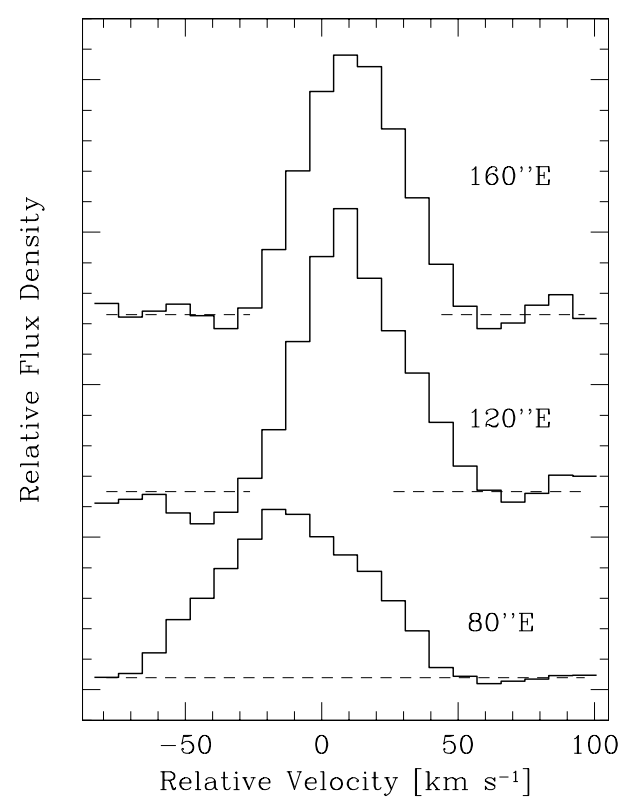

Fig. 8. Spectra at three locations along the plume. The spectral channel resolution is indicated by the histogram plot. Locations are labeled in arcsecs eastward from the Right Ascension of the host galaxy center. Velocities are measured with respect to $z=0.031355$. Baselines at zero flux density are indicated for each spectrum by dashed lines.

redshifted signal from VV 29c. The signature of rotation of $\mathrm{VV} 29 \mathrm{c}$ is also clear in this $L-V$ plane.

Figure 11 has plots of the integrated spectrum ("singledish") of the VV 29 system and the spectrum of the region surrounding the early-type companion galaxy 


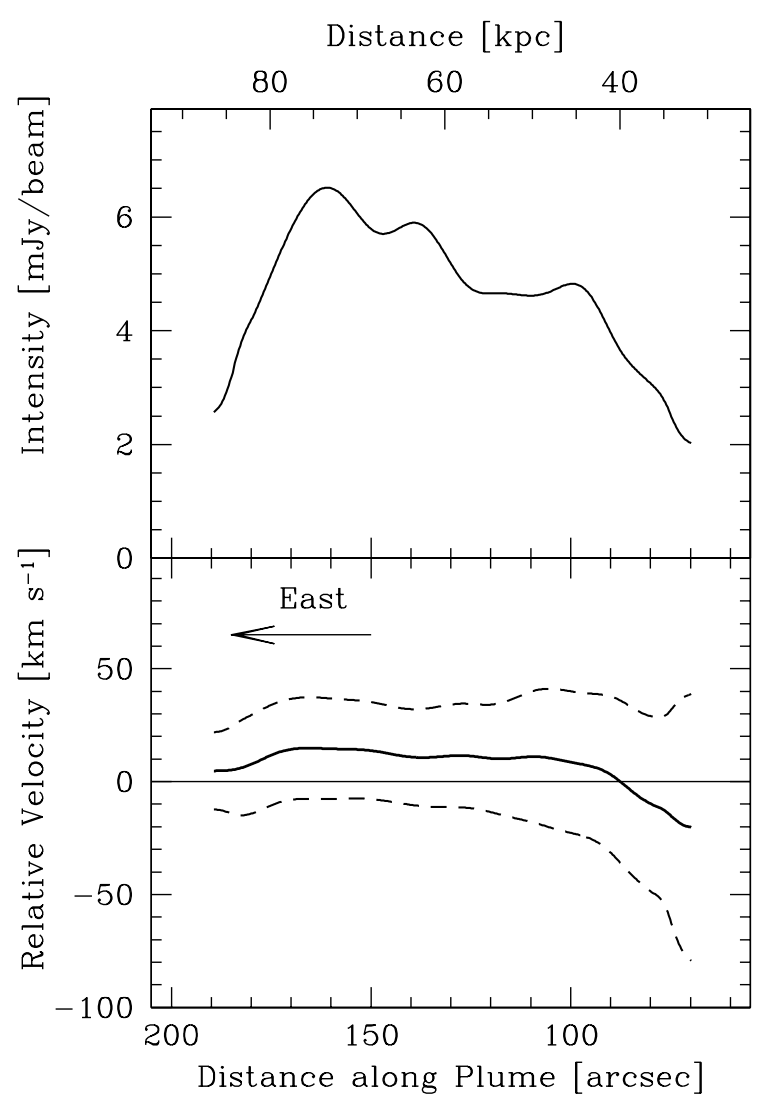

Fig. 9. Intensity, velocity centroid and velocity width from fitted Gaussian profiles along the plume. Velocity centroid (solid curve in lower panel) is indicated with respect to $z=$ 0.031355 , while velocity spread (measured at $F W H M$ ) is indicated by the dashed curves in the lower panel. A distance scale is drawn at the top assuming for Hubble Constant $H_{0}=$ $100 \mathrm{~km} \mathrm{~s}^{-1} \mathrm{Mpc}^{-1}$.

MCG 09-26-54. The very broad component emitted by the host galaxy is easy to miss in single-dish observations, as evidenced by the Nançay spectrum (Bottinelli et al. 1993), which recovered only the narrow profile of the plume. Figure 11 shows no evidence of any emission from MCG 09-26-54, implying an upper limit on its HI mass $\left(\Delta V / 50 \mathrm{~km} \mathrm{~s}^{-1}\right) \times 10^{8} h^{-2} M_{\odot}$ that is directly proportional to the assumed velocity width $\Delta V$.

\section{Discussion}

The WSRT observations reveal that the VV 29 system comprises at least three substantial components, a, b, and the newly identified VV 29c. Close inspection of deep optical images, in combination with clues from the $21 \mathrm{~cm}$ maps, reveals a still more detailed picture, which is summarized in the sketch in Fig. 13. Figure 12 shows a filtered, smoothed $g$-band INT image from Trentham et al. (2001), adjusted to high contrast to accentuate the dim features. The image shows a very low-level "counter-arm" to the west extending to the location of the HI detection of the "west plume" in Figs. 1 and 7. The deep image also has a clear vertical plume rising northward from the eastern side

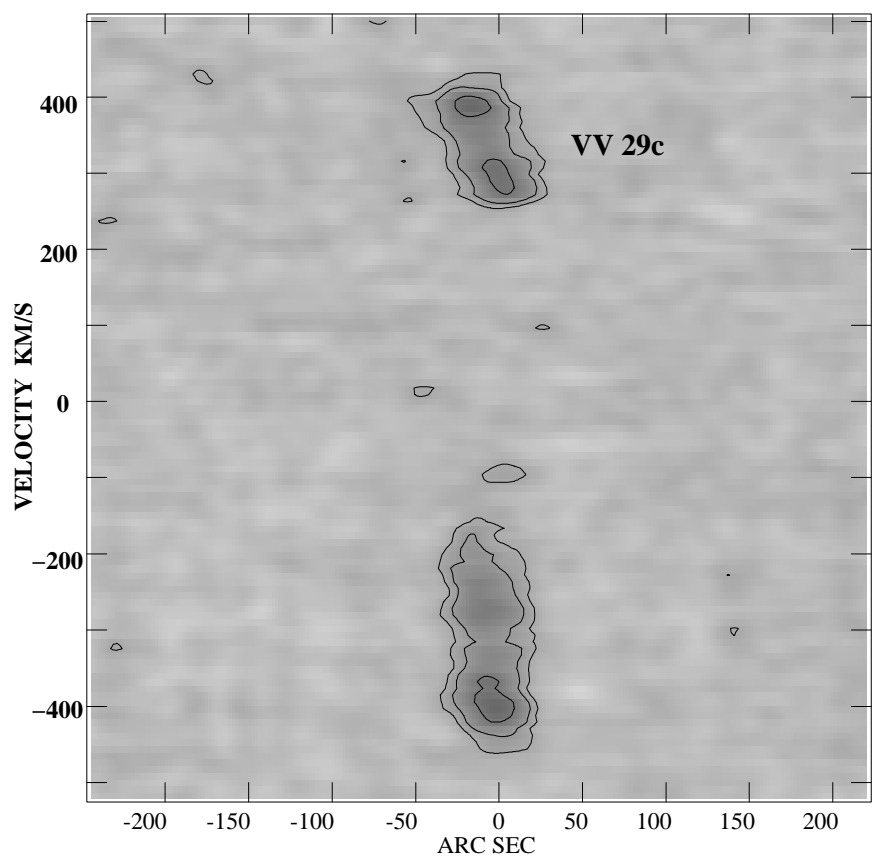

Fig. 10. Contours of HI brightness temperature in the distance-velocity plane along a line passing through the center of the blue dwarf VV 29c. The distance axis is a line of constant right ascension. The HI signal of the blue dwarf VV 29c falls in the upper region of the plot in the velocity range 250 to $420 \mathrm{~km} \mathrm{~s}^{-1}$. The zero of velocity is $z=0.031355$, and the origin of distance falls at $16^{\mathrm{h}} 06^{\mathrm{m}} 02^{\mathrm{s}} \cdot 0,55^{\circ} 25^{\prime} 32^{\prime \prime}(\mathrm{J} 2000)$. Contour levels are 1, 2, $4 \mathrm{~K}$.

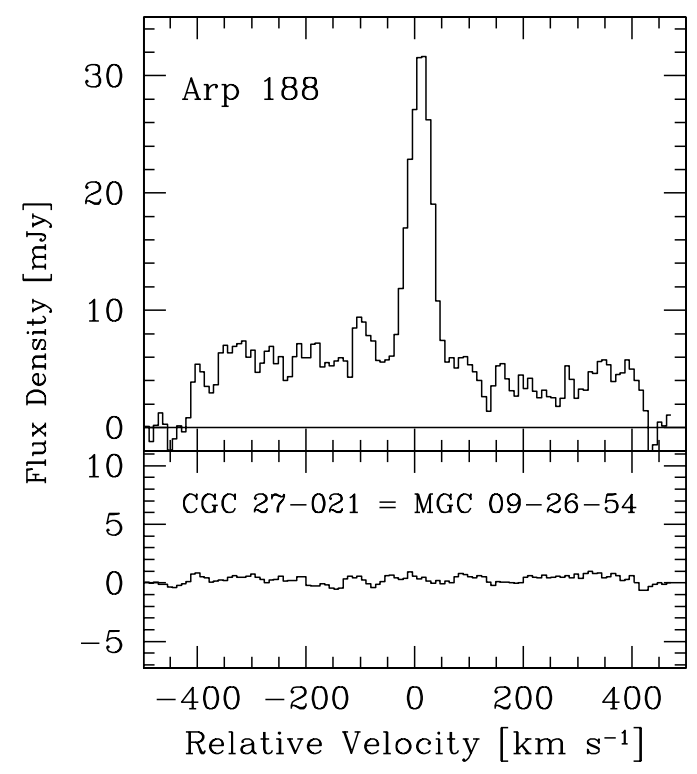

Fig. 11. Integrated flux density spectra for VV $29=\operatorname{Arp} 188=$ UGC 10214 and MCG 09-26-54. The integral for the VV 29 system (top panel) comes from summing the four spectra of Fig. 5. The MCG 09-26-54 spectrum (lower panel) comes from integrating the spectrum over the $52^{\prime \prime} \times 52^{\prime \prime}$ patch $(30 \mathrm{kpc} \times$ $30 \mathrm{kpc}$ ) centered on $16^{\mathrm{h}} 05^{\mathrm{m}} 48^{\mathrm{s}}, 55^{\circ} 21^{\prime} 53^{\prime \prime}(\mathrm{J} 2000)$, the celestial coordinates of MCG 09-26-54. The zero velocity corresponds to $z=0.031355$ (Heliocentric). 
of VV 29a, corresponding to the distorted HI contours in Fig. 1; comparison with lower contrast optical images indicates that the vertical plume attaches smoothly to a broad arc of faint optical emission to the south of VV 29a, which we interpret as a sort of partial polar ring or equivalent to the Magellanic Stream of the Local Group. Using $u_{-}, g-$, $r$-, and $i$-band INT images from Trentham et al. (2001), photometry measurements of several regions of the system reveal that the compact optical region at the position of VV29c and the long, thin central part of the plume are bluest. Several blue knots can be identified in the region of the plume, most likely corresponding to recently star forming regions. The compact blue source at the position of VV29c is embedded or, more likely, partly obscured by a significantly redder region that seems to be part of VV29a.

From the radio and optical data, it appears most likely that the newly identified VV 29c is located behind the host VV 29a and is receding from it, after having suffered a near collision with VV 29a. Any doubts might be resolved by optical and ultraviolet observations that would sense the dust extinction caused by the spiral arms of the VV 29a against VV 29c, if VV 29c is in the background.

The tidal plume VV 29b to the east displays the morphology and kinematics that Toomre \& Toomre (1972) described with restricted $N$-body calculations three decades ago. The gas appears to be both kinematically and morphologically connected to gas on the western side of the host, although the extreme western cloud/plume appears to represent the tip of a "counter-tail", stimulated in outskirts of VV 29a by the interaction. The bulk of the gas and the visible starlight in the long filament VV 29b are concentrated in the eastern plume at a distance of $\sim 40$ to $80 h^{-1} \mathrm{kpc}$ from the host.

Both Toomre \& Toomre (1972) and later Wallin \& Stuart (1992) examined a range of the collision parameters that specify the orientation of the axis of rotation relative to the orientation of orbital angular momentum of the interaction. The greatest disruption is inflicted on galaxies whose rotational angular momentum vector aligns with the orbital angular momentum vector. In the VV 29 case, we visualize the most massive galaxy, the VV 29a host that we view nearly edge-on, as the target galaxy. A fully intact, late-type, less-massive intruder is incident on the target, following a trajectory that carries it by VV 29a approximately in its disk plane, passing with the same sense of revolution as the target. This type of trajectory would be the most effective at raising a tail and countertail from the outskirts of the massive target VV29a. What makes VV 29 special in relation to simulated and other observed systems, like for example NGC 7252 (Dubinsky et al. 1996; Hibbard et al. 1994), is the compactness of the secondary galaxy and the high relative velocity.

Since the time of close encounter, VV 29c is likely to have traveled a distance that is similar to the distance traveled by the outer gas clouds in the VV 29b plume, so we estimate that it now lies roughly $100 \mathrm{kpc}$ behind VV 29a. The further implication is that from our

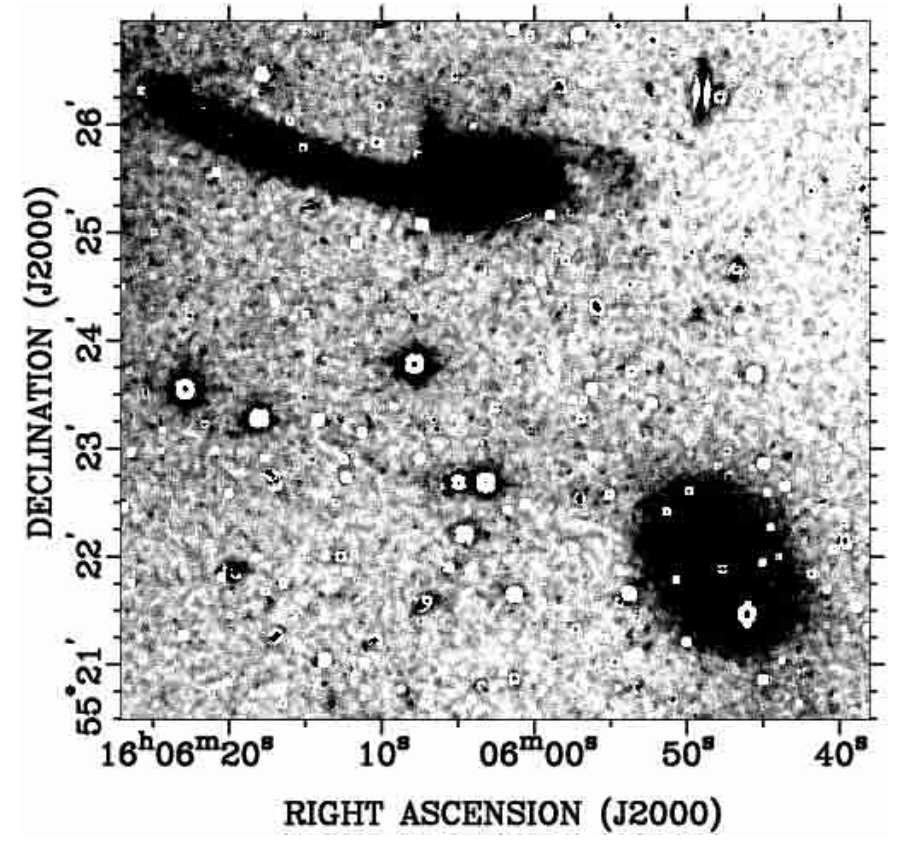

Fig. 12. Smoothed, filtered, high contrast $g$-band image of the VV 29 field, including the elliptical MCG 09-26-54 at lower right.

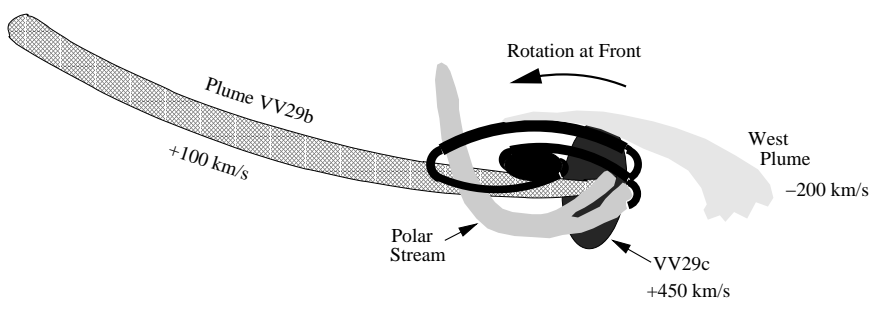

Fig. 13. Inventory of structures in the VV 29 system. Components are labeled with approximate line of sight velocities with respect to the systemic velocity of VV 29a. The eastern plume VV 29b, which passes behind the host galaxy VV 29a, may connect to the "upper" spiral arm of on the western side of VV 29a. The "polar stream" appears to start on the "lower" spiral arm and end by flipping up behind the eastern side of VV29 a, where it the distorts the HI contours in Fig. 1. The western plume is detected in HI and faintly visible in optical light. The blue dwarf galaxy VV 29c lies in the background.

viewpoint $\mathrm{VV}$ 29c passed from west to east in front of VV 29c and experienced an interaction that diverted its lateral motion to largely recessional motion with a slight westerly component to carry it back behind VV 29a. In this picture, we would expect VV 29c to eventually emerge from behind VV 29a on the western side. The interaction slings the VV 29b plume eastward and leaving it with a slight recessional velocity component with respect to the target VV 29a.

A plausible case can be made that we are viewing the product of tidal interaction between two normal galaxies. The dynamical components of the interaction are detectable through their baryon content - stars and hydrogen gas. 
Acknowledgements. The authors are grateful to R. Sancisi for valuable comments and to the staff of the Westerbork Telescope for their skill and dedication in bringing the upgraded Multi-Frequency Front Ends and the DZB correlation spectrometer systems into operation. The Westerbork Synthesis Radio Telescope (WSRT) is operated by the Netherlands Foundation for Research in Astronomy (NFRA) with financial support of the Netherlands Organization for Scientific Research (NWO). The deep optical images were made publically available through the Isaac Newton Groups' Wide Field Camera Survey Programme. The Isaac Newton Telescope is operated on the island of La Palma by the Isaac Newton Group in the Spanish Observatorio del Roque de los Muchachos of the Instituto de Astrofisica de Canarias. This research has made use of the NASA Astrophysics Data System (ADS) and the NASA/IPAC Extragalactic Database (NED) which is operated by the Jet Propulsion Laboratory, California Institute of Technology, under contract with the National Aeronautics and Space Administration.

\section{References}

Arp, H. C. 1962, Altas of Peculiar Galaxies (Pasadena: California Institute of Technology)

Arp, H. C. 1966, ApJS, 14, 1

Bottinelli, L., Durand, N., Fouque, P., et al. 1993, A\&AS, 102, 57
Dickey, J. M., Hanson, M. M., \& Helou, G. 1990, ApJ, 352, 522

Dubinsky, J., Mihos, J. C., \& Hernquist, L. 1996, ApJ, 462, 576

Hibbard, J. E., \& van Gorkom, J. H. 1996, AJ, 111, 655

Hibbard, J. E., Guhathakurta, P., van Gorkom, J. H., \& Schweizer, F. 1994, AJ, 107, 67

Higdon, J. L., \& Wallin, J. F. 2001, ApJ, submitted

Joseph, R. D., Meikle, W. P. S., Robertson, N. A., \& Wright, G. S. 1984, MNRAS, 209, 111

Schombert, J. M., Walling, J. F., \& Struck-Marcell, C. 1990, AJ, 99, 497

Shostak, G. S., \& van der Kruit, P. C. 1984, A\&A, 132, 20

van der Kruit, P. C., \& Shostak, G. S. 1982, A\&A, 105, 351

van der Kruit, P. C., \& Shostak, G. S. 1984, A\&A, 134, 258

van Zee, L., \& Bryant, J. 1999, ApJ, 118, 2183

Toomre, A., \& Toomre, J. 1972, ApJ, 178, 623

Toomre, A. 1977, in The Evolution of Galaxies and Stellar Populations, ed. B. M. Tinsley, \& R. B. Larson (Yale University, New Haven), 401

Trentham, N., Möller, O., \& Ramirez-Ruiz, E. 2001, MNRAS, 322,658

Vorontsov-Velyaminov, B. A. 1959, Atlas and Catalog of Interacting Galaxies, vol. 1 (Stenberg Institute, Moscow State University, Moscow)

Wallin, J. F., \& Stuart, B. V. 1992, ApJ, 399, 29

White, R. A., Bliton, M., Bhavsar, S. P., et al. 1999, AJ, 118, 2014 